\title{
Research on Extinguishing Performance of Efficient Fire Extinguishing Agent
}

\author{
Jiang Xiaogang ${ }^{a}$, Yuan Zhijiang ${ }^{b}$, Zheng Zhilin ${ }^{c}$, Zhai Yuting ${ }^{d}$ \\ Dept. of Navigation, Dalian Naval Academy, Dalian 116018, China \\ aforgething@163.com, ${ }^{\mathrm{b}}$ \\ yuanyr0531@163.com, ‘379105685@qq.com, di.music@163.com
}

\begin{abstract}
Water is very important as an extinguishing material. However, it is difficult to put out the fire for the long-range or complex environment because of vulnerable, dosage and low efficiency. In this paper, a kind of high-efficiency water-soluble fire extinguishing agent was developed. The results show that the effective firefighting distance was increased by more than $40 \%$ and firefighting time was reduced by more than $1 / 3$ when the fire extinguishing agent was used.
\end{abstract}

Keywords: Fire, Fire extinguishing agent, Water-soluble, Long-distance

\section{Introduction}

Long-distance, high-rise and complex environments have always been a difficult issue in the field of firefighting. In order to improve the fire away from the fire and fire efficiency, a lot of research work had been done. In the machinery and equipment, the water supply height was been increased to $140 \mathrm{~m}$, the maximum range of up to $1000 \mathrm{~m}[1]$, but the divergence of water would be caused. Water mist fire extinguishing as a high efficiency and low consumption of firefighting technology in many areas had also been applied which played a role in cooling based on the water mist particles[2], but can't be sprayed remotely. Li Zenghua used adding thickening additives in the water to reduce water loss and improve the rate of fire[3], but it would be easy to cause spraying difficulties, especially for long-range firefighting. In this paper, a new type of water-soluble high-performance fire extinguishing agent was developed through use of water-soluble surfactants and specific chemical reagents.

\section{Study on Fire Extinguishing Mechanism}

\subsection{Water Soluble Analysis of Firefighting Agents}

The components of the present extinguishing agent are water-soluble surfactants and one or several kinds of ammonium chloride, sodium chloride and sodium bicarbonate according to a certain proportion, wherein the water-soluble surfactant is sodium dodecyl sulfate, Polyethylene glycol, cocoal acid diethanolamide, or one or more. By referring to the relevant data, the above-mentioned components of the water solubility as shown in Table 1, it should be noted that the solubility in the table are standard atmospheric pressure $\left(20{ }^{\circ} \mathrm{C}\right)$ data [4]. 
Table 1 Fire extinguishing agent components of water solubility

\begin{tabular}{|c|c|}
\hline Name & Solubility $(\mathbf{m g} / \mathbf{m l})$ \\
\hline Ammonium chloride & 37.2 \\
\hline Sodium chloride & 36.0 \\
\hline Sodium bicarbonate & 9.7 \\
\hline Sodium dodecyl sulfate & 15 \\
\hline Polyethylene glycol & 50 \\
\hline Coconut acid diethanolamide & $5 \sim 10$ \\
\hline
\end{tabular}

As can be seen from the data in Table 1, the above chemical elements have a certain water solubility, according to the composition of the fire extinguishing agent and its solubility, so that the content of each component was less than its solubility, so as to ensure the dissolution of the fire agent.

\subsection{Fire Extinguishing Agent Fire Suppression Mechanism Analysis}

The role of the water-soluble surfactant in the fire extinguishing agent component is to increase the foaming effect of the fire extinguishing agent, which can cover the effect of suffocation and improve the fire extinguishing efficiency.

The chemical reaction of ammonium chloride after heating is as follows.

$$
\mathrm{NH}_{4} \mathrm{Cl} \triangleq \mathrm{NH}_{3} \uparrow+\mathrm{HCl}
$$

The use of the fire extinguishing agent, ammonium chloride heat easily generated ammonia, the deflagration phenomenon would be produced, and thereby the consumption of oxygen would be accelerated. Ammonia and oxygen reaction equation is as follows.

$$
4 \mathrm{NH}_{3}+3 \mathrm{O}_{2} \stackrel{\text { 燃烧 }}{\rightarrow} 2 \mathrm{~N}_{2} \uparrow+6 \mathrm{H}_{2} \mathrm{O}
$$

The chemical reaction of sodium bicarbonate after heating is as follows.

$$
2 \mathrm{NaHCO}_{3} \triangleq \mathrm{Na}_{2} \mathrm{CO}_{3}+\mathrm{H}_{2} \mathrm{O}+\mathrm{CO}_{2} \uparrow
$$

Sodium bicarbonate was heated to produce sodium carbonate, water and carbon dioxide, both of which have a good inhibitory effect on the fire. Sodium carbonate itself can be combined with hydrogen ions to produce the following reaction.

$$
\mathrm{Na}_{2} \mathrm{CO}_{3}+\mathrm{H}^{+} \rightarrow \mathrm{Na}^{+}+\mathrm{CO}_{2}+\mathrm{H}_{2} \mathrm{O}
$$

The free radicals required were not only consumed and the fire was inhabited. Sodium chloride is not easily decomposed by heat, but the reaction after ionization is as follows.

$$
\mathrm{NaCl} \rightarrow \stackrel{\text { 电离 }}{\rightarrow} \mathrm{Na}^{+}+\mathrm{Cl}^{-}
$$

Similarly, chloride ions generated by ionization of sodium chloride could be combined with hydrogen ions to form hydrogen chloride, but also played a role in reducing the free radicals required for combustion, which could inhibit combustion.

In summary, the nitrogen, carbon dioxide and water could be generated by the chemical reaction and a lot of oxygen would be consumed by ammonia reaction. In 
addition, some substances can consume the hydrogen radicals required for combustion, but also play a role in chemical inhibition.

\section{Experimental Study on Firefighting Performance}

\subsection{Experimental Study on Water Solubility of Fire Extinguishing Agent}

$500 \mathrm{ml}$ of the proposed fire extinguishing agent solution placed in the beaker which stood, in order to reduce the interference factors, the whole device stored in a relatively closed environment. The turbidity of the solution was measured at the same time every day by firing a beam of laser parallel through the bottom of the beaker where the precipitate was easily accumulated and measuring the laser intensity through the solution on the other side using a light meter. The experimental process had continued until the solution precipitation. The whole experimental procedure as described above, the experimental data shown in Table 2.

Table2. Extinguishing performance test data

\begin{tabular}{|c|c|c|c|c|c|c|c|c|c|c|c|}
\hline Time/day & 1 & 2 & 3 & 4 & 5 & 6 & 7 & 8 & 9 & $\ldots$ & 15 \\
\hline $\begin{array}{c}\text { Illumination } \\
\text { count value }\end{array}$ & 406 & 342 & 280 & 200 & 172 & 154 & 122 & 103 & 98 & $\ldots$ & 98 \\
\hline
\end{tabular}

From the experimental data, it is known that the illuminance count value gradually decreases with the passage of time, indicating that the extinguishing solution gradually precipitates, and after the 9th day, the illuminance count didn't change, indicating that the solution had reached the maximum.

The effluent rate was studied by contrast experiment. Two containers of the same specification were set up. The same piping and nozzle were used to spray the water and the mixed solution into the container. After stopping, the amount of the solution in the container was measured, and the effluent rate was obtained. According to the experimental needs, the nozzle diameter was set to $5 \mathrm{~mm}$, the pressure value of 0.2Mpa, spray with jet mode, through multiple experiments to collect data. It could be seen from the experimental data that the flow rate of the fire extinguishing agent solution is slightly lower than that of the ordinary fire water at the injection speed, but the difference is not significant.

\subsection{Experimental Study on Fire Extinguishing Efficiency}

In order to test the fire extinguishing agent of the extinguishing agent, a fire test was carried out using a long-term extinguishing agent. The $1000 \mathrm{ml}$ of diesel and $100 \mathrm{ml}$ of gasoline has been placed in the basin, the basin size of $30 \mathrm{~cm}$ in diameter. The experimental results were shown in Table 3.

Table3. Experimental data on oil fire extinguishing

\begin{tabular}{|c|c|c|}
\hline & Ordinary fire water & Extinguishing agent \\
\hline Firefighting time/s & 67 & 42 \\
\hline Water consumption/ml & 1470 & 760 \\
\hline
\end{tabular}

It can be seen from the experimental results, although the fire extinguishing agent flow had been reduced, but the fire time was greatly reduced, and consumption of less than ordinary fire water $1 / 2$. 
Firefighting distance test using ordinary water and fire extinguishing agent solution fire, the experimental distance as the benchmark, each away from the brazier $1 \mathrm{~m}$ to conduct a fire test, record the flow meter value and fire time. The experimental results are shown in Fig1.

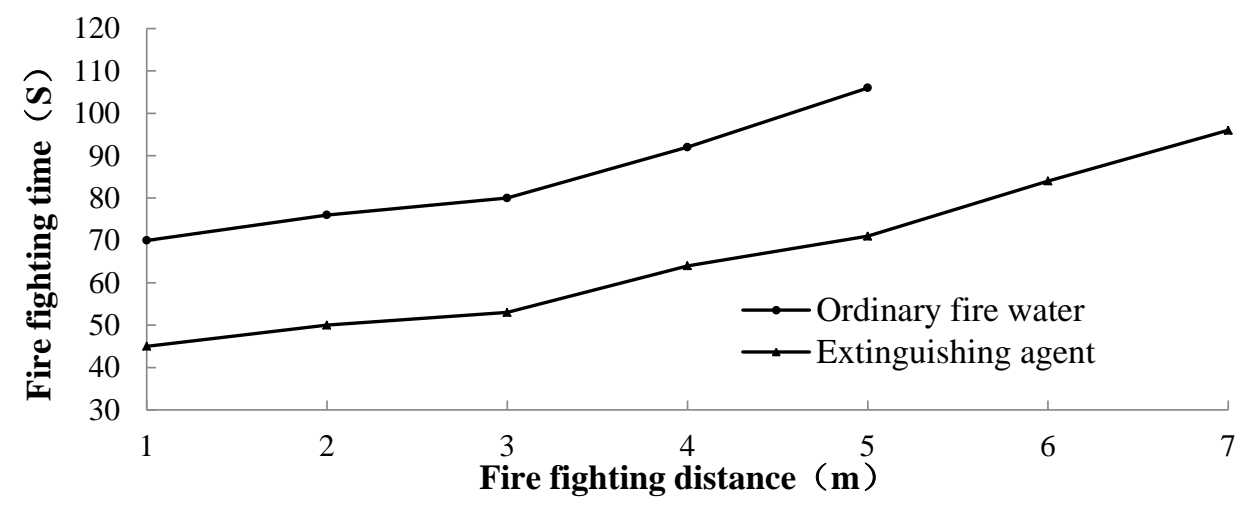

Fig.1 Experimental data curve of oil-fired fire extinguishing distance From the experimental data in Fig1, the fire extinguishing time and the water consumption of the common fire water and fire extinguishing solution were increasing as the firefighting distance increases, but the consumption of ordinary fire water was basically an extinguishing agent about twice the solution.

\section{Conclusion}

The water solubility of the special extinguishing solution meets the piping spray requirements and reduces the low solubility of the chemical formulation at the time of manufacture. Through the analysis of the corresponding chemical raw materials of the fire extinguishing agent solution, the fire extinguishing agent had the comprehensive fire extinguishing mechanism of cooling, choking and consuming free radicals. The experiments showed that the fire extinguishing agent with fire water fire away from the ordinary fire water more than $40 \%$, while the fire time to shorten more than $1 / 3$, the water consumption was only the original $1 / 2$.

\section{Acknowledgments}

This work was financially supported by Research and Development Fund Projects of Dalian Naval Academy.

\section{References}

[1] Xin Lei, Yu Wen-hua. Study on Water Pressure Loss of a Long-distance Water Supply-based Fire Extinguishing System, Forestry Machinery \& Woodworking Equipment, 2014(3):33-35.

[2] Chi Wei, Shi Kan, etc. A New Fire-fighting Technique Based on Special Fine Water Mists in Ships, Navigation of China, 2004(4),10-14.

[3] Li Zenghua, Zhou Shining. Study on the Properties and Extinguishing Mechanism of Viscous Water Extinguishant [J]. China Safety Science Journa,2003,13(9):41-43.

[4] Beijing Chemical Reagent Company. Chemical Reagent Standard Manual[M]. Beijing: chemical industry press, 2005. 